\title{
The Interpretation of Ethical Purpose of Urology
}

\author{
$\mathrm{Na}$ Wang \\ Department of Anesthesiology \\ The First Hospital of Jilin University \\ Changchun, China \\ wangna080613@163.com
}

\author{
Jinguo Wang* (corresponding author) \\ Department of Urology \\ The First Hospital of Jilin University \\ Changchun, China \\ wangjinguolily@163.com
}

\begin{abstract}
The development of society and modern medical science make the existing medical purpose face challenge, especially in urology which includes organ transplantation, andrology and reproduction which are all sensitive to ethics. The requirement of health is not only the basic need of human beings, but also the basic rights they have, which associated with life, ethics and human rights. People in the pursuit of self-value continuously create value for society, the personal value and social value in personal pursuit will reach unified implementation. On the base of the literature, we discuss the ethical purpose of urology.
\end{abstract} right

Keywords-interpretation; ethical purpose; urology; human

\section{INTRODUCTION}

Medical achievements of the 20th century are reviewed to bring the benefits of social progress, the threat of disease and early death has greatly reduced. But on the other hand also reveal some problems, namely with the advent of aging society, the application of medical high technology, per capita total health expenses rose too hard, people call it a "health crisis", and that will affect the sustainable development of the society. Citizens are not satisfied with medical service and medical staff's enthusiasm is not fully mobilized, the efficiency is not high, and because of a shortage of medical resources and implement some changes to "ration", essentially also brewing reform in these countries, there is a potential crisis [1]. In our country, for example, in some parts of the situation of the medical expense rise too hard existing more than ten years, many enterprises have unbearable, western countries have encountered problems in our country is just beginning. Are not only the United States and other developed countries face such a problem we also faced with the problem?

\section{ETHICAL PURPOSE OF UROLOGY}

System was to improve the health level of the people played an important role, has made remarkable achievements. The world bank and the world health organization delivered a investigation report, the Chinese developed "cooperative medical treatment" (refers to the ownership of the rural collective economy, farmers rely on the collective strength to start production brigade clinic, cultivating medical and farmers "barefoot doctors", for basic and commonly used drugs, medical devices provide basically free health services for rural residents) called "the only model for developing countries to solve health funds".
Before China's ministry of health minister in China health development and decision-making, the "scientific" and "authority" book, very proudly said, "since the world health organization (who) along with the director-general Dr. Mahler, have positive to developing countries recommended" China's rural health work experience. With 19 June 2000, however, the world health organization published the 53rd health assembly (world health report 2000 -health systems: improve performance. In the report, the world health organization set up three general goals for the first time to evaluate the health system performance. The three overall goals are: 1, "the improvement of health degree"; 2, the reactivity of the crowd expectations; 3, "and" F "for financial burden. In the 191 member countries around the world after the performance to make a quantitative assessment of the health system, the report on the health performance ranked in these countries." the poor "in our country health burden accounted for the proportion of its revenue is far higher than the" rich "health burden accounted for the proportion of their income. And people has always been regarded as" the gap between rich and poor is great ", "distribution was unfair," the most populous country, India is ranked 43, far more than China and among the world top. In just 20 years, China's health performance should get two evaluation is the opposite of the world health organization. This conclusion is startling.

\section{THE CURRENT STATUS OF ETHICAL PURPOSE}

Of course, the report was met by some countries including China. The reason is that the evaluation system is not scientific, comprehensive information collection is not enough, and the sample bias deviation factors leads to the conclusion [2]. However, the ostrich to type this report is not desirable, on the contrary, we should treat this report as a warning. In fact, even without the support of adjustable I data, we can truly feel I trapped in the aspect of health "financial burden fairness" of the serious problems, for example, quite a number of high earners in China, as well as ordinary residents, still enjoying the state financial subsidies under the low charge standard, even take part in social medical insurance, the insurance premium is paid according to the same pay scale.

In the cities, especially in the southeast coastal areas, have begun to appear some medical and health services are faced with the situation similar to the western developed countries. In these areas, disease composition is very different from the Midwest rural, due to the extension of life expectancy, the aging of the population process very quickly. Health preserves mainly tube disease and other chronic diseases. Health care 
service pattern in these places is the solution to follow the western developed countries, thus drew a series of contradictions: one is the key of the reality of medical and health services and health needs of the broad masses to ask. Second, countries with limited health into the development of medical and health services demand the contradiction between the increasing. Three is a social enterprise, individual limited financial resources and health care costs, the economic burden to love more and more unbearable, the contradiction of the past is over the disease, is now a look down on disease. Four is big medical center (hospital) and the contradiction of small and medium-sized medical institutions, many small and mediumsized hospitals in large hospital the advantage of high technology and equipment, many personnel before is close to close. Five is the medical service quality is not high, the landslides and the masses of medical ethics and ask the contradiction of require thoughtful and careful services. Six is excess some aspects of medical service and the contradiction in the shortage of primary health care.

At present, countries in I trapped large and medium-sized cities, the medical burden of the enterprise, for any hospital management, more and broad worker opinion, is the performance of the current health crisis in our country.

That is worth pointing out, in order to alleviate this contradiction and some hospitals were forced to go to the market, an operator in an attempt to find way out of the health care crisis from market. To this end, some hospitals to carry out the examination and treatment of high and new technology in great quantities, a large number of using drugs and imported drugs, improve the charge standard, opening up new services and fees, hospital beds, etc. These measures also indeed received an immediate effect many hospitals have higher income, medical personnel treatment also improved. But, this a series of practice as a result, not only further stimulate the medical consumption, and increased social and personal medical burden, thus eventually threaten the health care service its own survival [5].

\section{COMPARISON WITH OTHER COUNTRIES}

The dangers of the problem is that not only a serious violation of the principle of health financing ability to pay and the principle of fairness, but also intensified the "poverty due to illness" and "poor return disease" vicious circle, make "the poor poorer, the rich get richer", to eventually lead to society [3]. Although this is not news, but it is the research center under the state council in 2005 at the end of July of "China ten years reform basic not successful" after "remains a critical" again and again and again. Research center under the state council declared health reform failed after rectified time, health information follow: officials deny "doom". Have unit many times sent out as soon as the approval of the state council has set the new medical reform, etc. An elite have propped up market of health care, says is the international practice. Someone said "we don't India's low level of universal free medical care the union hospital is not everyone can enter".

In middle and western regions of our country, especially those in rural areas, the main contradiction is still the underserved. In these areas, threatening the health of major infectious diseases, infectious diseases and parasitic diseases. Combined with the market economy environment, the influence of doctors, nurses are scarce, the masses of these areas are about disease problem become more prominent [4]. If you do not take effective measures, the medical supplies to meet the objective needs of contradictions will further highlight.

In view of the above "health crisis", the last two decades the United States and many home in policy and management level, a wide variety of reform measures, test and so on, but these reforms are often only receive short-term effects, but deep-seated problems difficult to solve.

\section{THE PURPOSE ACCEPTED BY THE SOCIETY}

Medical philosophers thought after the second world war in many infectious diseases, epidemic under control, cure and prevention of disease more easily obtained the very good control effect, with the rapid development of basic medicine and clinical medicine, the development of emergency medicine, transplant hospital, new operation method, therapeutic and diagnostic methods of development made amazing achievements, many people think the purpose of medical treatment is the treatment of diseases, the pursuit of cure and cure, and prevent death as a divine purpose. This purpose, although not found in dictionary or written expression, but it is accepted by people [6].

This purpose in medicine acute disease, infectious diseases as the main object, is very certain, but some recession against chronic diseases and disorders is pale, when it is difficult to cure chronic diseases, cure and the cost of the larger emergency medical rescue means for recession patients dying rescue is difficult to appear miracle "coming back to life". And chronic diseases, recessionary disease prevention before and make the necessary response. As a result, it lost the light of the early 20 th century in preventive medicine. After the discussion and research, they have proposed four medical purposes to prevent illness and injury promote and maintain a healthy. Remove the pain and suffering caused by disease. Care (Care) and treatment for the disease, the Care of an incurable disease. Avoid early death, and pursuit of a peaceful death. Prevention is the aim of medical cure, safeguard human health. The fundamental purpose of medicine, since the ancient times is the guide of medical staff, so far no outdated. But the medicine in the process of its thousands of years of development, its total target although there is no change, but in different periods of specific goals are different. At the beginning of the medical form, for example, to pain, I'm afraid the purpose of medicine is just in addition to the disease such as prevention of other diseases, reduce mortality, I'm afraid not. Later, with the development of medicine and medical people want to see on the rise, the contents of medical purpose is increasingly rich and diverse.

The modern medicine with the aim to make a new evaluation, thus led to a discussion of medical purpose. The Chinese medical association medical ethics society held in November 1994, the second session of the council for the first time, carefully study the medical purpose discuss, think this is a meaningful and closely associated with medical ethics important subject, and therefore decided to "medical purpose, 
the quality of life, medical ethics" as the theme at the eighth conference on medical ethics.

This time in October 1995 conference received more than 200 papers, with the medical profession all aspects of the waste heat in Beijing and with the six experts were invited to do a report. People spoke, meeting the medical ethics, society and medical purpose did a survey of more than 1000 public. This is a cause widespread reaction of the meeting. "The health" prominently reported not only in the first meeting, and then to discuss meeting made a special interview. Journal of medicine and philosophy, medicine and social, display "journal of medical university journals, such as have some of the dozens of the conference papers. For presentation to the eighth national academic meetings to discuss medical ethics of "several problems about adjusting medical purpose and service mode of the research outline", learn to the secretariat to continue the meeting after the meeting for advice, and have modified according to comments, male issued in 1996, in order to cause more in-depth discussions, and will submit to the relevant leaders of the state council and the ministry of health, the outline of the Chinese medical association, and caused the attention of the relevant responsible comrades.

Why medical purpose in today's so everyone's attention? This is from the current medical purpose and the situation of the face. The overall goal of the current medical and past a long time, people are not so far apart. But now medical specific goal is on the "three rates", namely the incidence, mortality, life expectancy rate per person. Incidence of as little as possible, the death rate is lower, the better, life as long as possible, and to be an indicator to measure health work achievement. For example the concise British encyclopedia in each country's health work is introduced, and emphasis on mortality, life expectancy, the national average has a number of doctors and beds and other indicators. This was the right which these indicators indeed reflect a country the level of health work. But now, it seems, from the whole situation faced by the current, the health work target mainly concentrated on the "three rates", put the main objects of health work in "disease" and "patient", there are some drawbacks, need to study and adjust.

Because of the health care industry is everyone needs, a person want to survival and development, is implied behind people's life and health. Maslow's hierarchy of needs also pointed out that human beings itself health concerns and needs, not only is the most basic human needs, is also should have the basic human rights and freedom, the relevant to life ethics of human rights.

Maslow believes that physiological needs are the most primitive, the most basic needs, such as eating, clothing, housing, medical treatment and so on. If not satisfied, have life risk. That is to say, it is at the bottom of the strongest inevitably needs and promotes people's strong driving force for action. Clearly, the physiological needs of self and the protection of ethnic significance give priority to with hunger and thirst, is the need of human individual and essential in order to survive. When there are many a person needs, such as a lack of food, safety, and love at the same time, always hungry need to possess the biggest advantage of the lack of food, it shows that when controlled by a human physiological needs, then other all need (security needs, social needs, respect for the needs and self-actualization needs) were pushed into the background. The need of human there are two different value systems, one kind is rising along the biological spectrum direction gradually weaken instinct or impulse, referred to as low-level needs and physiological needs. One kind is along with the evolution and the potential of emerging or need, known as advanced needs. Are lurking in the five different levels of need, but at different times of various needs urgently degree is different [7]. The most urgent need is the main reason of motivating action and motivation.

\section{CONCLUSIONS}

The needs of the people from outside of meet gradually to inner get satisfaction. At a high level from the custom of the need to fully meet the needs of the low level must be properly. After the needs of the low level basically satisfied, it will reduce the incentive function of its dominant position will no longer continue, high-level will need to replace it as the main original driving behavior. Some need to once meet, cannot be the cause of the inspire behavior, and been replaced with other needs. From a certain sense, we can say that individual's "prime" demand is the demand of society as a whole individual low level is constitutes the motive force of social development, the satisfaction of everyone in order to pursue their lowest hierarchy of needs or better satisfied and contribute their own strength, the intricacies of the dialectical combination of the innumerable, driving the development of the society. People in the pursuit of self-value continuously create value for society, the personal value and social value in personal pursuit I reached unified implementation.

\section{REFERENCES}

[1] Aaron Ciechanover. The Personalized Medicine Revolution: Are We Going to Cure All Diseases and at What Price?[A].2014

[2] Ya-juan Li. On the Relationship of the Law-related Education and the Rule-of-Law Society[A]. Proceedings of 2014 2nd International Conference on Economics and Social Science(ICESS 2014 V61)[C]. 2014

[3] Qi Wang. Analysis of the Internal Relations between Morality and Law[A]. Proceedings of 2013 Fourth International Conference on Education and Sports Education(ESE 2013 V12)[C]. 2013

[4] Gazmararian JA, Baker DW, Williams MV, Parker RM, Scott TL, Green DC, Fehrenbach SN, Ren J, Koplan JP. Health literacy among Medicare enrollees in a managed care organization. JAMA: the journal of the American Medical Association. 1999

[5] Bass M J, Buck C, Turner L, Dickie G, Pratt G, Robinson H C. The physician's actions and the outcome of illness in family practice. The Journal of family practice. 1986

[6] McClellan W. The physician and patient education: a review. Patient Education and Counseling. 1986

[7] Anne Underwood, Jerry Adler. When Cultures Clash: A growing medical challenge that doctors and patients often can't communicate. Newsweek. 